\title{
STUDIES OF VISCERAL PAIN: MEASUREMENTS OF STIMULUS INTENSITY AND DURATION ASSOCIATED WITH THE ONSET OF PAIN IN ESOPHAGUS, ILEUM AND COLON
}

\author{
By MARTIN LIPKIN ${ }^{1}$ AND MARVIN H. SLEISENGER \\ (From the Department of Medicine, New York Hospital-Cornell Medical Center, \\ New York, N. Y.)
}

(Submitted for publication December 5, 1956; accepted September 12, 1957)

Past studies of visceral pain have generally been concerned with qualitative aspects of pain production. However, the occurrence of visceral pain has not been studied in terms as quantitative as those used to describe the onset of cutaneous pain. As a result, physiologic concepts of visceral pain which might be based on such measurements have not been derived.

In previous experiments, pain has been produced in the gastrointestinal tract by the application of a distending force to the hollow viscus. A commonly used method of making such studies is that of placing in the viscus a balloon attached to a manometer. In previous studies the magnitude of applied pressure has been identified with the intensity of stimulus required to produce pain. A great variability has been found in the stimulus intensity which resulted in pain in the same and different subjects under the experimental conditions used (1-3). However, there has been no study involving simultaneous measurements of stimulus intensity and duration associated with the onset of pain, as performed in the present experiments.

The objectives of the present investigation were: 1) to produce pain in the gastrointestinal tract with a stimulus, certain characteristics of which could be accurately determined and controlled; 2) to study the relationship between intensity and duration of stimulus required to produce pain; and 3) to assess the reproducibility of these measurements in the same and in different individuals.

\section{METHODS}

The apparatus used in these experiments is shown diagramatically in Figure 1 . Chamber A was a cylindrical water tank 45 centimeters in diameter, which had

\footnotetext{
1 Formerly, United States Pubic Health Service Post-
} doctorate Research Fellow. a cross-sectional area of $1,590 \mathrm{~cm}^{2}$ at the fluid surface. By means of firm plastic tubing, chamber $\mathrm{A}$ was connected to a latex balloon, C. Chamber B was an additional water reservoir, and $\mathrm{D}$ and $\mathrm{E}$ were mechanically operated valves. Alternate connection of balloon and chambers A or B could be effected by means of the valves. During an experimental determination, the balloon and chamber $\mathrm{A}$ were connected. Because of the large cross-sectional area at the fluid surface in chamber $\mathrm{A}$, the height of water did not measurably change. The diameter of the tubing was $0.75 \mathrm{~cm}$., and the entire length of tubing was $290 \mathrm{~cm}$. With the fluid surface in chamber A $100 \mathrm{~cm}$. above the end of the tubing, water flowed from the tubing at a rate of $49 \mathrm{ml}$. per second. The resistance of the balloons to distention was also studied in vitro. Measurements were made of pressures within the balloons at various levels of distention. The maximum pressure attained from expansion of the balloons in vitro was $11 \mathrm{~cm}$. of water. In the present experiments, the intensity of the stimulus was measured as the height of water in chamber $A$. The relationship between the pressure, $\mathrm{P}_{\mathrm{o}}$, determined by the height of water in chamber $A$, and the pressure, $P$, applied to the viscus, may be given by equation (I) in which

$$
P=P_{0}-f_{1}\left(\frac{\pi}{2} D l \frac{d D}{d t}\right)-f_{2}(D)
$$

The function $f_{1}$ describes the pressure decline in the tubing, and is equal to the rate of volume expansion of the balloon, where $\mathrm{D}$ and 1 are respectively the diameter and length of the balloon. The function $f_{2}$ describes the small pressure differential needed to expand the balloon. In this series of experiments relationships between the intensity of stimulus, $\mathrm{P}_{0}$, and the time to onset of pain were studied. Relationships between $f_{1}$ and $f_{2}$ and the onset of pain were not studied. The principle utilized in these studies of visceral pain, in which the externally applied stimulus was maintained constant during an experimental determination, was the same as that used in studies of cutaneous pain $(4,5)$.

Identical water tanks, valves and tubing were used for all experiments, with a new condom balloon of the same brand in each experiment. Forty-five male and six female subjects ranging in age from 20 to 53 years were studied. Five of the subjects were normal physicians and medical students, and 46 were hospital patients without evidence of gastrointestinal disease or functional 


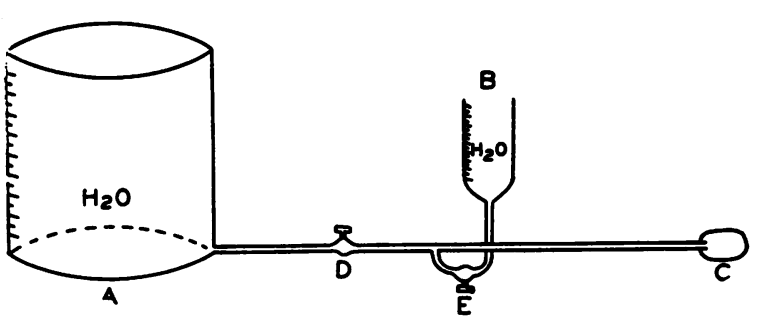

Fig. 1. Diagram of Apparatus Used in These EXPERIMENTS

Chamber A was a large water tank, connected by firm tubing to a latex balloon, C. Chamber B was an additional water reservoir; $D$ and $E$ were mechanically operated valves by means of which alternate connection of the balloon and chamber A or B could be made.

disorder. The orientation of the subjects to the experimental procedure was similar to that utilized in studies of cutaneous pain $(4,5)$. Each subject was carefully instructed regarding the purpose and method of the study. The subjects were asked to signal at the onset of a painful sensation, and then were asked to describe it in terms of quality, position, intensity, duration and radiation. The pain threshold was defined as the time of onset of the earliest perceptible sensation of pain. The description of the sensation given by each subject was carefully noted, and in all instances closely corresponded to classic descriptions of visceral pain (6). Several studies of the colon were performed with the authors as subjects, and in these instances the onset of pain could be clearly defined within the time range noted by the other subjects at the various pressures.

In each experiment, a balloon was attached to the distal end of the tubing. The tube and balloon were then filled with water. With experience at this procedure, it was possible to manipulate the tube and balloon so that air bubbles were eliminated. The tube and balloon were also sufficiently transparent so that small air bubbles could be seen if present and removed. A clamp was applied to the proximal end of the tube to prevent the loss of water from the tube and introduction of air bubbles during intubation. The tube and balloon were then introduced to the desired position. In experiments on the esophagus, the subject swallowed the tube aided by the sipping of iced water, and the tube and balloon were positioned so that the tip was just above the cardioesophageal junction. Studies of the small intestine were performed in patients who had ileostomies for ulcerative colitis. All patients in this category had been operated on more than two years prior to this procedure and had no symptoms or signs of disease of the ileum. Intubation was performed without any prior preparation. The tube and balloon were introduced into the stoma, and passed $20 \mathrm{~cm}$. For studies of the sigmoid colon, patients were prepared with a low Fleet enema (hypertonic phosphate solution). A proctoscope was passed to 12 to $15 \mathrm{~cm}$., the tube and balloon inserted, and the proctoscope

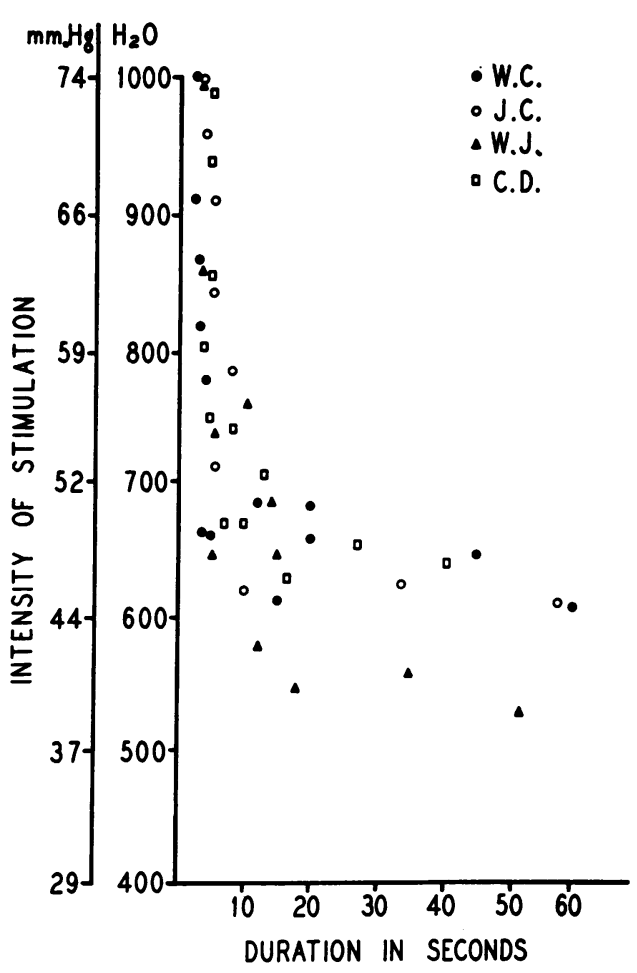

Fig. 2. Relationship of Stimulus Intensity and Duration Required to Elictt Pain, Following DisTENTION OF ESOPHAGUS

The intensity of stimulus is shown in $\mathrm{mm}$. of $\mathrm{Hg}$ and mm. of $\mathrm{H}_{2} \mathrm{O}$.

removed. The tube and balloon were then passed gradually to 30 to $35 \mathrm{~cm}$. The subjects were supine during all experimental determinations. The position of the tube was determined fluoroscopically with the aid of radiopaque thread tied to the end of the tube.

When the tube and balloon were in the desired position, the clamp was removed and the entire water filled system connected, again making sure to avoid the introduction of air bubbles. Initially, the balloon and distal tubing were connected to the additional water reservoir (chamber B, Figure 1). The height of water in chamber B was set at the same height as the anterior abdominal wall. After an interval of 15 minutes, chamber $\mathrm{B}$ was disconnected and chamber A suddenly connected to the system. Operation of the valves was not visible to the subjects. At the onset of pain, the subject relayed a signal and the time was noted. Chamber A was immediately disconnected and chamber B connected to the tubing and balloon, resulting in a flow of water from the balloon into chamber $B$. In all instances, the sensation of pain subsided with the flow of water from the balloon. The outflow of water into chamber B was also measured. After an interval of five minutes, the determination was repeated. 


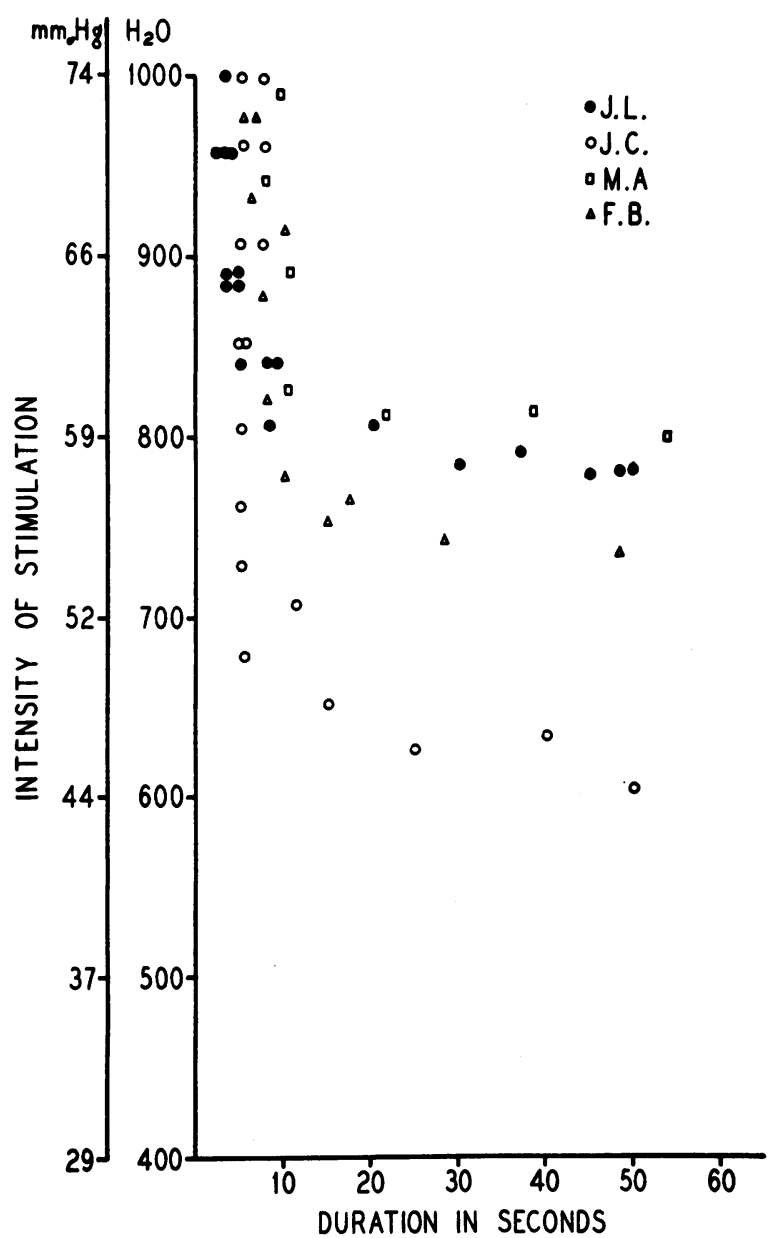

Fig. 3. Relationship of Intensity and Duration of Stimulus Required to Elicit Pain Following DisTENTION OF ILEUM

In the first series of experiments the relationship of the intensity and duration of the stimulus to onset of pain threshold was studied. Four experiments with esophageal intubation were performed on four subjects. Four experiments were performed on four patients with ileostomies, and seven determinations on the sigmoid colon of six subjects. In the last group, four of the subjects were doctors and medical students.

In the second series of experiments, $P_{0}$ was set at 100 $\mathrm{cm}$. of water, and the duration to pain threshold was studied in the sigmoid colon. The frequency distribution of 171 observations made on 37 subjects was noted. Repeated observations were also made on four subjects over a period of three to four months.

\section{RESULTS}

In Figures 2, 3, and 4, the relationship of intensity of stimulation to duration measured for threshold pain originating in the esophagus, ileum and colon are shown. When the intensity of stimulation was large, pain was usually the first sensation perceived. In measurements with low intensity stimulation accompanied by longer duration prior to the onset of pain, the subjects often noted the appearance of a sensation of pressure prior to pain. In all instances, the sensation of pressure was related to a larger area than was pain. From within the area in which pressure was perceived, the sensation of pain arose, and was described as aching in quality. Esophageal pain was referred to the midline at a level of the second to fifth thoracic segments; pain from the lower ileum at a level of the eighth to tenth thoracic segments and colonic pain to the eleventh and twelfth thoracic segments within 3 inches to the right and 3 inches to the left of the midline. In Figures 2 through 4 it is seen that an increase in intensity of stimulation is accompanied by a decrease in duration required for the response. In addition, greater variation in duration is noted at lower intensities of stimulation than at higher intensities. The minimum time to pain threshold was 2 seconds for the esophagus, 3 seconds for the ileum, and 2 seconds for the colon. In experiments on the esophagus, if no pain appeared within 40 to 60 seconds after the application of low intensity stimulation, none appeared thereafter. The corresponding durations were 50 to 55 seconds for ileal measurements, and 40 to 60 seconds for the colon. The minimal intensity of stimulation required to elicit esophageal pain was 520 to $620 \mathrm{~mm} . \mathrm{H}_{2} \mathrm{O}$. The corresponding intensity for experiments on the ileum was $600 \mathrm{~mm}$. $\mathrm{H}_{2} \mathrm{O}$ for subject J. C. However, the minimal intensity required for the other subjects was higher, at 740 to $800 \mathrm{~mm} . \mathrm{H}_{2} \mathrm{O}$. In experiments on the colon, the minimal intensity was 540 to $670 \mathrm{~mm}$. $\mathrm{H}_{2} \mathrm{O}$ with greater variability at lower intensities of stimulation than was present in determinations on esophagus and ileum.

In the second series of determinations, the duration to pain threshold was studied in the sigmoid colon of 37 subjects at a single intensity of applied stimulation. Figure 5 shows the total compilation of these pain threshold measurements. The mean pain threshold value was 6.6 seconds, with a standard deviation of \pm 3.6 seconds. The results of repeated studies made on four subjects are shown in Figure 6. The time thresholds 
measured for these subjects show little variation when repeated at intervals of several weeks.

\section{DISCUSSION}

\section{Characteristics of stimulus}

Studies of pain sensation carried out in the past have stressed the importance of understanding many characteristics concerned with evaluation of stimulus and response (4). Certain principles derived from this past experience have been followed in the present study. In order to evaluate adequately the occurrence of the sensation of pain, the stimulus should meet the following requirements :

The stimulus used under experimental conditions should be very similar to the noxious stimulus which causes naturally occurring pain. The characteristics of the stimulus should be measured, and should be controlled to a fine degree. It should, therefore, be possible to maintain the externally applied stimulus constant over a given duration and to reproduce easily any stimulus intensity. The stimulus should produce the sensation of pain in such a manner that the sensation is clearly identified.

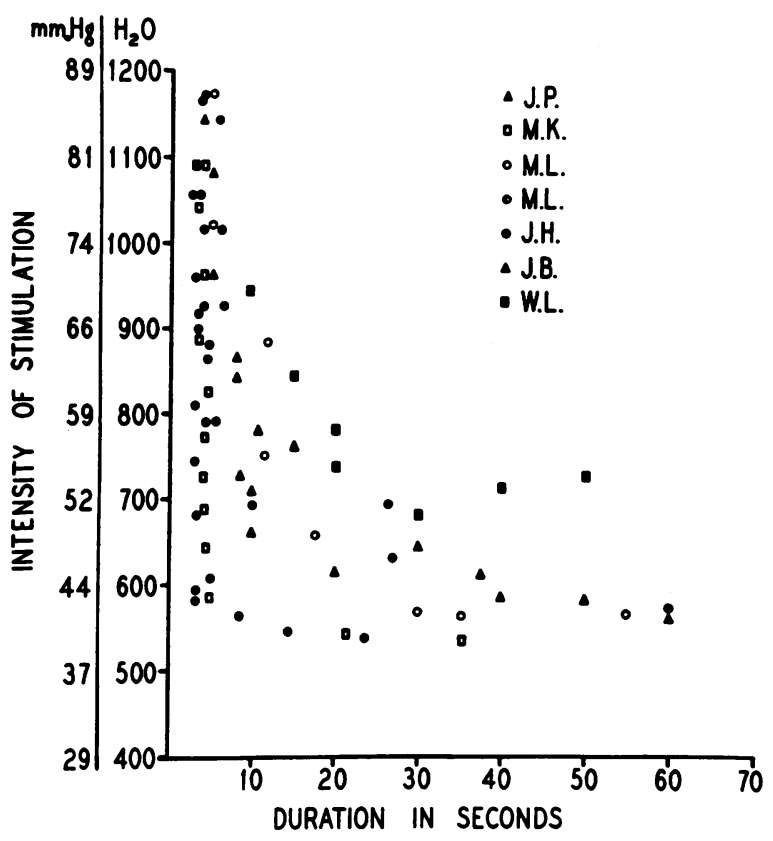

Fig. 4. Relationship of Intensity and Duration of Stimulus Required to Elicit Pain, Following Distention of Sigmoid Colon

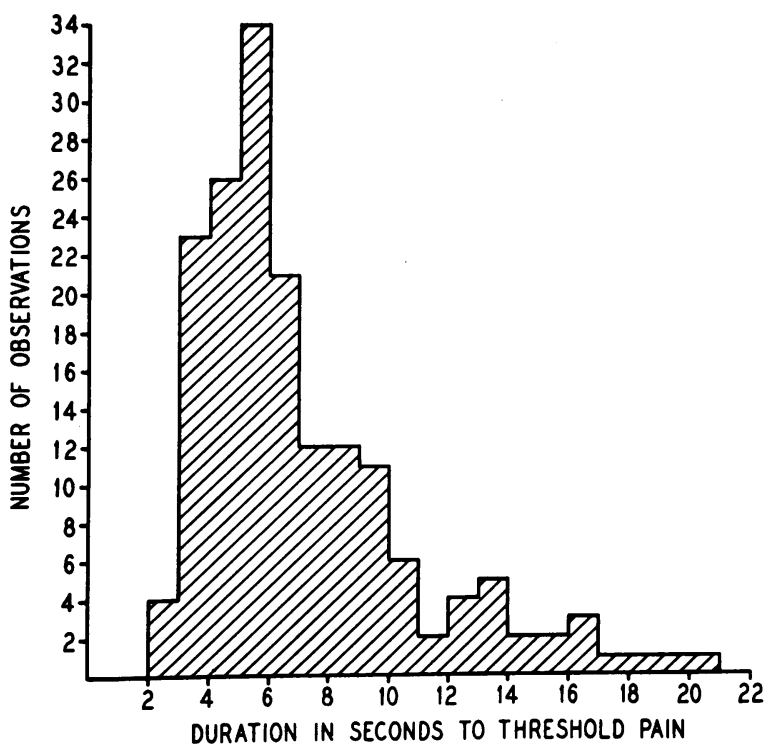

Fig. 5. Frequency Distribution of Durations to Pain Threshold Following Distention of Sigmoid Colon, at a Single Intensity of Applied Stimulation

\section{Intensity-duration measurements}

Studies of the responses of a wide variety of living tissues to many types of stimulation have revealed a characteristic relationship between the intensity of the stimulus and the duration required to produce a given response, namely, that a greater intensity of stimulation is associated with a shorter duration required to elicit the response (7). The same relation of stimulus intensity and duration has been shown in the production of cutaneous pain (8). The fact that the onset of visceral pain appears to follow the same general rule with respect to stimulus intensity and duration is of particular interest. Intensity-duration curves have not previously been demonstrated for the onset of visceral pain.

As in other studies of intensity-duration curves $(4,9)$, the term "rheobase" may be used to denote the intensity of stimulation below which the response will not occur. The term "utilization time" may be used to denote the minimum time of stimulation effective for rheobase intensity. In these experiments, the rheobase for 3 subjects was in a higher range ( 740 to $800 \mathrm{~mm} . \mathrm{H}_{2} \mathrm{O}$ ) than for the other 11 subjects ( 520 to $700 \mathrm{~mm} . \mathrm{H}_{2} \mathrm{O}$ ). The values for higher rheobase occurred following distention of ileum in the group with previous ul- 


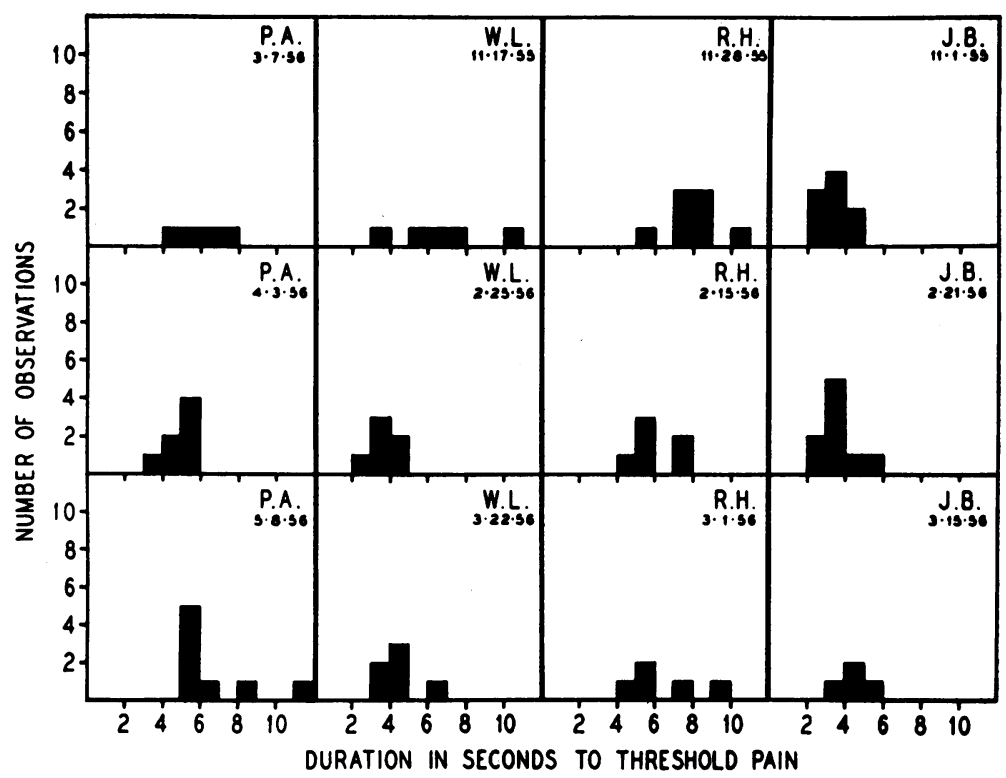

Fig. 6. Duration to Threshold Pain Following Distention of Sigmoid Colon, at a Single Intensity of Stimulation

Repeated observations made on four subjects. $P_{0}$ was set at $100 \mathrm{~cm}$. of water. The duration to pain threshold for each subject is shown on the abscissa. The number of observations for each of the three experiments performed on each subject is shown on the ordinate.

cerative colitis and colonic resection. Differences in rheobase values should reflect variations in the individual tissues, since the apparatus was the same in all determinations. The differences in rheobase might be caused by variations in innervation of the segments of a viscus, or by variations in resistance to distention present in the viscus. In addition, different balloon lengths present after distention in segments of varying resistances could have resulted in stimulation of unequal lengths of intestine. The relationship of the rate of distention of the viscus to the onset of pain cannot be stated with certainty. The possibility that a lower rheobase is associated with an increase in the rate of distention of the viscus is supported by the observation that a greater intensity of stimulation causes an increased rate of expansion of the balloon in vitro, and an earlier onset of pain in vivo. An earlier onset of pain at a given stimulus intensity would result in a lower rheobase. However, increased intramural tension in the viscus associated with a decreased rate of distention might also result in an earlier onset of pain and a lower rheobase.

There are certain corrections which should be made to the externally applied pressure measurements in order to evaluate the actual pressure applied to the wall of the intestine. These corrections relate to the pressure drop in the tubing, occurring when large rates of flow exist, and to the pressure differential associated with the elastic expansion of the balloon as described in equation (I). If the actual pressure applied to the viscus were known, then a more accurate evaluation of intensity-duration relationships would be possible. However, in the present preliminary study, as in studies of cutaneous pain $(5,8)$, the intensity of the externally applied stimulus was measured, rather than measurement of the actual stimulus applied to the nerve endings. It is believed that despite these omissions, the intensity-duration relationship noted is correct. Thus, a correction for pressure drop would not be expected to decrease the vertical spread of the data; a general downward shift of the points shown would increase the ratio of maximum to minimum pressure.

\section{Comparisons of visceral and cutaneous pain}

In studies of tissue response to stimulation, it has been noted that a tissue may react to a stimu- 
lus in such a way as to oppose alteration of the normal status of the tissue $(7,10)$. This characteristic which has been termed "accommodation" or "adaptation" may be a significant part of the response to low intensity stimulation. It has also been noted in studies of the response of cutaneous tissue to thermal stimulation utilized to elicit pain. In the latter case, it has been shown that when normal skin is exposed to thermal radiation, cutaneous blood flow to the area is increased. The thermal energy available to induce a rise in skin temperature is thus reduced, and an abnormal rise in skin temperature is opposed (11). The occurrence of cutaneous pain is related to an inability of increased blood flow to prevent a temperature rise, and if the skin temperature rises to $45^{\circ} \mathrm{C}$., pain occurs. The onset of pain in this instance has been shown to be related to the onset of tissue damage and is believed to be caused by protein denaturation along the nerve endings (12).

In studies of cutaneous pain, it has been possible to study the reproducibility of the time threshold to pain, and at the same time to evaluate the response of accommodation to the stimulus. It has thus been shown that with high intensity stimulation cutaneous blood flow does not change, and the variation in time threshold is very small. With low intensity stimulation, however, cutaneous blood flow does increase, and the time threshold is more variable (11).

Studies relating the onset of visceral pain to stimulus intensity and to local tissue changes have thus far not been carried out as precisely as the manner in which cutaneous pain has been studied. However, on the basis of observations made here and in other studies, certain similarities regarding the production of visceral and cutaneous pain may be noted. Lower intensities of stimulation in both instances are associated with greater variation in the time threshold for pain. It is also known that lower intensities of stimulation permit the tissues to undergo various changes of accommodation or adaptation before the onset of pain, which high intensities of stimulation do not. Thus, after the application of pressure to a viscus, great increase in diameter of the viscus may occur (1). This may involve changes in length and tension of smooth muscle fibers (13). In the occurrence of visceral pain, it may be postulated that differences in accommodation result in greater variation in the time threshold for pain than occurs with cutaneous pain. In addition, the fact that pain is more likely and accommodation less likely to occur with high intensity stimulation, makes it appear that changes of accommodation act to oppose the onset of pain. It would seem that the changes which take place in the viscus in response to pressure might function in the same manner as changes in cutaneous blood flow after thermal stimulation. That is, they would prevent the stimulus from causing the onset of pain. In the case of thermal cutaneous stimulation, the onset of pain is also associated with the production of tissue damage. Whether the onset of visceral pain is also associated with the onset of tissue damage is not known at present.

\section{SUMMARY}

The relationship between the intensity and duration of stimulus required to produce pain in the esophagus, ileum and colon has been studied in 51 subjects. Intubation of the organ was performed with balloon and catheter. The balloon-catheter system was attached to a water chamber characterized by a large cross-sectional area at the fluid surface. The intensity of the stimulus was measured as the height of water in the chamber, and the height of water did not measurably change during each experimental determination. The duration to the earliest perceptible sensation of pain was measured.

A relationship of stimulus intensity and duration was found for the onset of visceral pain, which is similar to that shown for cutaneous pain. Intensity-duration curves plotted for studies of the three organs were very similar. Differences in rheobase values were believed to be due to properties intrinsic in the tissues and to variations in the rate of distention of the segment of viscus.

The mean duration to colonic pain threshold in 37 subjects studied at a single stimulus intensity was 6.6 seconds with a standard deviation of \pm 3.6 seconds. Repeated observations made on four subjects with several weeks between experiments revealed little variation in pain threshold for each subject.

Comparison of the characteristics of intensityduration curves obtained from measurements of visceral and cutaneous pain revealed the possi- 
bility that changes of local tissue accommodation prevent the onset of both varieties of pain.

\section{ACKNOWLEDGMENTS}

The authors would like to thank Dr. Thomas P. Almy and Dr. James D. Hardy for their review of this work, and Dr. William F. Nickel for assistance in intubation of the ileostomy patients.

\section{REFERENCES}

1. Wolf, S. G., and Wolff, H. G., Human Gastric Function, 2nd. ed. New York, Oxford University Press, 1947.

2. Chapman, W. P., and Jones, C. M., Variations in cutaneous and visceral pain sensitivity in normal subjects. J. Clin. Invest., 1944, 23, 81.

3. Gaensler, E. A., Quantitative determination of the visceral pain threshold in man. J. Clin. Invest., 1951, 30, 406.

4. Hardy, J. D., Wolff, H. G., and Goodell, H., Pain Sensations and Reactions. Baltimore, Williams and Wilkins Co., 1952.

5. Hardy, J. D., Wolff, H. G., and Goodell, H., Studies on pain. A new method for measuring pain threshold: Observations on spatial summation of pain. J. Clin. Invest., 1940, 19, 649.

6. Jones, C. M., Digestive Tract Pain, Diagnosis and Treatment. New York, Macmillan Co., 1938.

7. Heilbrunn, L. V., An Outline of General Physiology, 2nd. ed. Philadelphia, W. B. Saunders Co., 1943.

8. Bigelow, N., Harrison, I., Goodell, H., and Wolff, H. G., Studies on pain: Quantitative measurements of two pain sensations of the skin, with reference to the nature of the "hyperalgesia of peripheral neuritis." J. Clin. Invest., 1945, 24, 503.

9. Rushton, W. A. H., Time factor in electrical excitation. Biol. Rev. \& Bull., Proc. Cambridge Philosophical Soc., 1935, 10, 1.

10. Fulton, J. F., A Textbook of Physiology, 17th ed. Philadelphia, W. B. Saunders Co., 1955, p. 19.

11. Lipkin, M., and Hardy, J. D., Measurement of some thermal properties of human tissues. J. Applied Physiol., 1954, 7, 212.

12. Lipkin, M., Bailey, O., and Hardy, J. D., Effect of ultraviolet irradiation upon the cutaneous pain threshold. J. Applied Physiol., 1955, 7, 683.

13. Heinbecker, P., and Bishop, G. H., The mechanism of painful sensations in Sensation: Its Mechanisms and Disturbances. A. Research Nerv. \& Ment. Dis., Proc. Baltimore, Williams \& Wilkins C.o., 1935, p. 226. 